\title{
Mendelian randomization indicates that TNF is not causally associated with Alzheimer's disease
}

Shea J Andrews ${ }^{\mathrm{a}^{*}}$ \& Alison Goate ${ }^{\mathrm{a}}$

a Ronald M. Loeb Center for Alzheimer's disease, Department of Neuroscience, Icahn School of Medicine at Mount Sinai, New York, NY, USA

${ }^{*}$ Correspondence to: Shea Andrews, The Icahn School of Medicine at Mount Sinai, 1 Gustave L. Levy Place, New York, NY 10029.

Tel: +1-212-659-8632; E-mail: shea.andrews@mssm.edu 


\section{Abstract}

2 INTRODUCTION: Epidemiological research has suggested that inhibition of tumor necrosis

3 factor (TNF)- $\alpha$ in patients with rheumatoid arthritis (RA) reduces the overall risk of Alzheimer's

4 disease (AD). TNF- $a$ antagonists have been suggested as a potential treatment for AD.

5 METHODS: We used a two-sample Mendelian randomization design to examine the causal

6 relationship between blood TNF expression, serum TNF-a levels, and RA on AD risk.

7 RESULTS: Our results do not support a causal relationship between TNF expression, serum

8 TNF- $\alpha$ levels or RA on AD risk.

9 DISCUSSION: These results suggest that TNF- $\alpha$ antagonists are unlikely to reduce the risk of 10 AD.

12 Keywords: Alzheiemr's disease; Tumor necrosis factor; TNF; rheumatoid arthritis; Mendelian

\section{Randomization}




\section{Introduction}

17 Alzheimer's disease $(A D)$ is a debilitating neurological condition that is characterized by a

18 progressive deterioration in cognitive function and concomitant functional decline. While the

19 classical neuropathological hallmarks of $A D$ are the deposition of neuritic plaques and 20 neurofibrillary tangles, increasing evidence has highlighted the role of neuroinflammation and 21 microglial innate immune response in AD pathogenesis [1].

Chronic systemic inflammation may also be associated with an increased risk of developing

24 dementia [2], with higher levels of serum proinflammatory cytokines being reported in patients

25 with AD [3]. Chronic systemic inflammation is characterized by the production of the

26 proinflammatory cytokine tumor necrosis factor $a($ TNF- $\alpha$ ) from macrophages. TNF- $\alpha$ is involved

27 in the pathogenesis of chronic autoimmune disorders such as rheumatoid arthritis (RA), but also

28 plays a role in activation of the central innate immune response, including in microglial cells [4].

29 Inflammation represents a potential means of modifying AD pathogenesis, with the link between

30 peripheral inflammation, TNF- $\alpha$ and neuroinflammation suggesting that TNF- $\alpha$ inhibition may

31 reduce the risk of $A D$.

33 AD was found to be more prevalent in RA patients in a nested case-control study of 8.5 million

34 commercially insured adults. Additionally, it was observed that the risk of AD was lower among

35 RA patients who had been exposed to the TNF-a inhibitor etanercept, suggesting that anti-TNF 36 therapy with etanercept could be a potential treatment for $A D$ [5]. The potential role of 37 etanercept as a treatment for $A D$ has gained further media exposure from a recent Washington 38 Post report that analysis conducted by Pfizer, which holds the patent for etanercept outside of 39 the USA, observed a similar decrease in risk of AD in RA patients exposed to etanercept based 40 on insurance claim data [6]. Pfizer, however, elected not to proceed with a clinical trial which 
41 was estimated to cost $\$ 80$ million, according to critics, because etanercept was reaching the

42 end of its patent life, though Pfizer denies this was a factor [6].

43

44 While randomized control trials (RCTs) can be reliably used for estimating causal effects, they

45 are expensive to conduct and not all drug targets can be tested in an RCT framework due to

46 ethical considerations or the time-scale involved may be prohibitive. A novel method for

47 estimating the causal effect of drug targets on a disease outcome is Mendelian randomization

48 (MR). MR is a method that estimates the causal effect of an exposure on an outcome by using

49 genetic variants as a proxy for the exposure administered as an intervention in an RCT [7]. MR

50 is analogous to an RCT due to the random allocation of genotypes from parents to offspring at

51 conception (randomization in an RCT) and is thus not affected by reverse causation or

52 confounding variables. In the context of drug development, genetic variants are selected that

53 mimic the action of a drug target, with one allele associated with an altered gene or protein

54 expression (the drug in an RCT) to that of a neutral allele that serves as a reference (the

55 placebo in an RCT) [7]. If the altered genetic allele is associated with a pathway that is

56 causative of the disease, the MR study will detect a change in the clinical outcome. A drug

57 target that has a causative effect on disease is a potential target for drug development, whereas

58 the reverse is true if it is not causative.

59

60 In this study, we use Mendelian randomization to evaluate if RA, TNF gene expression and

61 TNF-a levels are causally related to AD risk.

\section{Methods}

\subsection{Instrument Selection}

64 We obtained cis-eQTL data derived from whole blood for TNF expression from the eQTLGen

65 project $(n=31,684)$ [8], pQTL data derived from whole blood TNF-a levels $(n=8,293)$ [9], and 
genome-wide significant SNPs for RA from a previous GWAS meta-analysis $(14,361$ RA cases

67 and 43,923 controls) [10]. To obtain independent SNPs, linkage disequilibrium clumping was

68 performed by excluding SNPs that had an $r 2>0.001$ with another variant with a smaller $p$-value

69 association within $1000 \mathrm{~kb}$ use a reference panel of European individuals from 1000 Genomes

70 Project (phase 3). Three independent eQTLs for TNF, six nominally significant $(p<5 e-6)$

71 independent TNF-a pQTLs, 56 independent SNPs for RA were selected for analysis. As the

72 effect sizes of the eQTLs were not available in the summary data, the effect sizes were

73 estimated from z-statistics as previously described [11].

75 The GWAS summary data for AD were from the most recent meta-analysis conducted by the

76 International Genomics of Alzheimer's Project comprised of 21,982 cases and 41,944

77 cognitively normal controls (Stage 1 discovery) [12]. The SNPs corresponding to the TNF

78 eQTLs, TNF-a pQTLs, and RA SNPs were extracted from the AD GWAS and were harmonized.

\subsection{Mendelian Randomization Analysis}

81 We used two-sample MR to estimate the causal effect of TNF expression, TNF-a levels, and RA

82 on $A D$. For each variant, we calculated an instrumental variable ratio estimate by dividing the

83 SNP-exposure by SNP-outcome and coefficients. An overall estimate of the causal effect was

84 calculated by combining the individual SNP estimates in a fixed-effects meta-analysis using an

85 inverse-variance weighted (IVW) approach [13]. In order to account for potential violations of the

86 assumptions underlying the IVW analysis, we conducted a sensitivity analysis using MR-Egger

87 regression, which allows all variants to be subject to direct effects [13] and the Weighted

88 Median Estimator (WME), which takes the median effect of all available variants, allowing 50\%

89 of variants to exhibit horizontal pleiotropy [13]. Heterogeneity was tested using Cochran's $Q$ 90 statistic [13]. 
91 The proportion of variance in the exposure explained by each instrument were calculated as

92 previously described [14]. Power calculations were conducted using the mRnd power

93 calculation tool [15]. All statistical analyses were conducted using $R$ version 3.5.2, with

94 Mendelian randomization analysis was performed using the 'TwoSampleMR' package [13].

\section{Results}

97 The selected instruments for TNF expression, TNF-a levels, and RA risk explained 5.93\% (F = $98285), 1.68 \%(F=23.6)$, and $19.2 \%(F=247)$ of the variance respectively. Given a sample size 99 of 63,926 with the proportion of cases equal to 0.34 , this study was adequately powered to detect an OR of any AD of 1.1 for TNF expression, 1.19 for TNF- $\alpha$ levels and 1.055 for RA.

There was no evidence of a causal association of TNF expression, TNF- $a$ levels or RA on AD risk in the IVW, WME, or MR-Egger regression analyses (Table 2). Similarly, there was no causal association for the individual TNF eQTLs. There was evidence of heterogeneity $(Q=$ 84.8, $d f=54, p=0.00472)$ in RA analysis, but not for the $\operatorname{TNF}(Q=3.46, d f=2, p=0.177)$ or TNF-a $(Q=2.78, d f=5, p=0.733)$ analysis.

\section{Discussion}

109 This study examined the causal association of blood TNF expression, serum TNF-a levels and

110 RA with AD risk using Mendelian randomization. Despite adequate statistical power to detect an

111 effect, we do not find any evidence that increased TNF expression, TNF-a levels or RA risk are

112 causally associated with increased AD risk. These results suggest that TNF- $\alpha$ antagonists, such 113 as etanercept, are unlikely to reduce the risk of $A D$. 
115 Incidence of $A D$ was reported to be lower in RA patients in a meta-analysis of 10 studies,

116 however, an MR analysis conducted using an earlier AD GWAS also found no causal effect of

117 RA on AD [16]. While animal studies of AD models suggest that TNF- $\alpha$ inhibition ameliorates

118 AD-related pathology, only a few human studies have been conducted [17]. An open-label

119 clinical trial conducted in mild-severe AD patients $(n=15)$ found that perispinal extrathecal

120 administration of etanercept was associated with significant improvement in cognitive function

121 [18]. In contrast, a double-blind study of etanercept conducted in patients with mild-moderate

$122 \mathrm{AD}(\mathrm{n}=41)$ over a 24-week period, found that subcutaneous administration of etanercept

123 showed no effect on cognitive, functional or behavioral assessments [19].

125 The results of this study should be interpreted in conjunction with its limitations. First, the analysis conducted here was restricted to the expression of TNF mRNA in whole blood, the tissue in which the largest eQTL studies have been conducted to date. Analysis in additional tissues may implicate TNF expression as a causal risk factor, however, the sample sizes available for other tissues are 30x smaller than that of whole blood and thus have considerably reduced power [20]. Second, the TNF-a GWAS did not contain any genome-wide significant

131 hits, as such, we used nominally significant hits which can result in the inclusion of weak 132 instruments and bias results towards the null. Finally, these MR estimates represent the effect 133 of lifelong exposure to increased TNF expression or TNF-a levels, while drugs generally have 134 shorter periods of exposure, and may not distinguish between critical periods of exposure [7].

136 In conclusion, this Mendelian randomization analysis does not support a causal effect of 137 increased blood TNF expression, serum TNF-a levels or RA risk on the risk of AD. These 138 results suggest that, in contrast to recent reports, TNF- $\alpha$ antagonists are unlikely to result in 139 decreased risk of $A D$. Furthermore, this study highlights how incorporating genetic data into the 
140 drug discovery process using Mendelian randomization can improve the drug discovery

141 process.

142

\section{$143 \quad$ Funding}

144 SJA and AMG were supported by the JPB Foundation (http://www.jpbfoundation.org).

145

\section{Conflicts of Interest}

147 SJA has no conflicts of interest to declare.

148 AMG served on the scientific advisory board for Denali Therapeutics from 2015-2018. She has

149 also served as a consultant for Biogen, Cognition Therapeutics, AbbVie, Pfizer, GSK, Eisai and 150 Illumina. 
[1] Mhatre SD, Tsai CA, Rubin AJ, James ML, Andreasson KI. Microglial malfunction: the third rail in the development of Alzheimer's disease. Trends Neurosci 2015;38:621-36.

[2] Wyss-Coray T. Inflammation in Alzheimer disease: driving force, bystander or beneficial response? Nat Med 2006;12:1005-15.

[3] Lai KSP, Liu CS, Rau A, Lanctôt KL, Köhler CA, Pakosh M, et al. Peripheral inflammatory markers in Alzheimer's disease: a systematic review and meta-analysis of 175 studies. J Neurol Neurosurg Psychiatry 2017;88:876-82.

[4] Perry VH, Cunningham C, Holmes $\mathrm{C}$. Systemic infections and inflammation affect chronic neurodegeneration. Nat Rev Immunol 2007;7:161-7.

[5] Chou RC, Kane M, Ghimire S, Gautam S, Gui J. Treatment for Rheumatoid Arthritis and Risk of Alzheimer's Disease: A Nested Case-Control Analysis. CNS Drugs 2016;30:111120.

[6] Rowland C. Pfizer had clues its blockbuster drug could prevent Alzheimer's. Why didn't it tell the world? The Washington Post 2019.

[7] Walker VM, Davey Smith G, Davies NM, Martin RM. Mendelian randomization: a novel approach for the prediction of adverse drug events and drug repurposing opportunities. Int J Epidemiol 2017;46:2078-89.

[8] Võsa U, Claringbould A, Westra H-J, Bonder MJ, Deelen P, Zeng B, et al. Unraveling the polygenic architecture of complex traits using blood eQTL metaanalysis. bioRxiv 2018:447367. doi:10.1101/447367.

[9] Ahola-Olli AV, Würtz P, Havulinna AS, Aalto K, Pitkänen N, Lehtimäki T, et al. Genomewide Association Study Identifies 27 Loci Influencing Concentrations of Circulating Cytokines and Growth Factors. Am J Hum Genet 2017;100:40-50.

[10] Okada Y, Wu D, Trynka G, Raj T, Terao C, Ikari K, et al. Genetics of rheumatoid arthritis contributes to biology and drug discovery. Nature 2014;506:376-81.

[11] Zhu Z, Zhang F, Hu H, Bakshi A, Robinson MR, Powell JE, et al. Integration of summary data from GWAS and eQTL studies predicts complex trait gene targets. Nat Genet 2016;48:481-7.

[12] Kunkle BW, Grenier-Boley B, Sims R, Bis JC, Damotte V, Naj AC, et al. Genetic metaanalysis of diagnosed Alzheimer's disease identifies new risk loci and implicates $A \beta$, tau, immunity and lipid processing. Nat Genet 2019. doi:10.1038/s41588-019-0358-2.

[13] Hemani G, Zheng J, Elsworth B, Wade KH, Haberland V, Baird D, et al. The MR-Base platform supports systematic causal inference across the human phenome. Elife 2018;7. doi:10.7554/eLife.34408.

[14] Shim H, Chasman DI, Smith JD, Mora S, Ridker PM, Nickerson DA, et al. A multivariate genome-wide association analysis of 10 LDL subfractions, and their response to statin treatment, in 1868 Caucasians. PLoS One 2015;10:e0120758.

[15] Brion M-JA, Shakhbazov K, Visscher PM. Calculating statistical power in Mendelian randomization studies. Int J Epidemiol 2013;42:1497-501.

[16] Policicchio S, Ahmad AN, Powell JF, Proitsi P. Rheumatoid arthritis and risk for Alzheimer's disease: a systematic review and meta-analysis and a Mendelian Randomization study. Sci Rep 2017;7:12861.

[17] Ekert JO, Gould RL, Reynolds G, Howard RJ. TNF alpha inhibitors in Alzheimer's disease: A systematic review. Int J Geriatr Psychiatry 2018;33:688-94.

[18] Tobinick E, Gross H, Weinberger A, Cohen H. TNF-alpha modulation for treatment of Alzheimer's disease: a 6-month pilot study. MedGenMed 2006;8:25.

[19] Butchart J, Brook L, Hopkins V, Teeling J, Püntener U, Culliford D, et al. Etanercept in Alzheimer disease: A randomized, placebo-controlled, double-blind, phase 2 trial. 
Neurology 2015;84:2161-8.

[20] GTEx Consortium, Laboratory, Data Analysis \&Coordinating Center (LDACC)—Analysis Working Group, Statistical Methods groups-Analysis Working Group, Enhancing GTEx (eGTEx) groups, NIH Common Fund, $\mathrm{NIH} / \mathrm{NCl}$, et al. Genetic effects on gene expression across human tissues. Nature 2017;550:204-13. 
Table 1: Causal effect of TNF expression, TNF- $\alpha$ levels and rheumatoid arthritis risk on

212 AD

\begin{tabular}{llll}
\hline & $\boldsymbol{\beta}(\mathbf{s e})$ & OR $(95 \% \mathrm{Cl})$ & $\mathbf{p}$ \\
\hline TNF - LOAD & & & \\
rs1121800 & $0.06(0.05)$ & $1.07(0.97,1.17)$ & 0.20 \\
rs72855945 & $0.17(0.19)$ & $1.19(0.83,1.72)$ & 0.35 \\
rs9469017 & $-0.24(0.17)$ & $0.79(0.57,1.1)$ & 0.16 \\
IVW & $0.05(0.05)$ & $1.05(0.96,1.15)$ & 0.30 \\
WME & $0.06(0.05)$ & $1.06(0.97,1.17)$ & 0.21 \\
MR Egger & $0.1(0.26)$ & $1.11(0.67,1.83)$ & 0.76 \\
TNF- $\alpha$ - LOAD & & & \\
IVW & $-0.03(0.04)$ & $0.97(0.89,1.06)$ & 0.52 \\
WME & $-0.03(0.06)$ & $0.97(0.87,1.08)$ & 0.58 \\
MR Egger & $-0.07(0.07)$ & $0.93(0.81,1.06)$ & 0.34 \\
RA - LOAD & & & \\
IVW & $-0.01(0.01)$ & $0.99(0.97,1.01)$ & 0.37 \\
WME & $0.01(0.02)$ & $1.01(0.97,1.06)$ & 0.48 \\
MR Egger & $-0.02(0.03)$ & $0.98(0.93,1.03)$ & 0.51 \\
\hline
\end{tabular}

214 TNF: Tumor necrosis factor mRNA expression; TNF-a: Serum tumor necrosis factor-a levels;

215 RA: rheumatoid arthritis 